University of South Carolina

Scholar Commons

$10-17-2007$

\title{
Structural Characterization Using the Multiple Scattering Effects in Grazing-Incidence Small-Angle X-Ray Scattering
}

\author{
Byeongdu Lee \\ Argonne National Laboratory \\ Chieh-Tsung Lo \\ Argonne National Laboratory \\ P. Thiyagarajan \\ Argonne National Laboratory \\ Dong R. Lee \\ Pohang Accelerator Laboratory \\ Zhongwei Niu \\ University of South Carolina - Columbia, niu.z@mail.chem.sc.edu
}

See next page for additional authors

Follow this and additional works at: https://scholarcommons.sc.edu/chem_facpub

Part of the Chemistry Commons

\footnotetext{
Publication Info

Published in Journal of Applied Crystallography, Volume 41, Issue 1, 2007, pages 134-142.

(C) Journal of Applied Crystallography 2007, International Union of Crystallography.
}

This Article is brought to you by the Chemistry and Biochemistry, Department of at Scholar Commons. It has been accepted for inclusion in Faculty Publications by an authorized administrator of Scholar Commons. For more information, please contact digres@mailbox.sc.edu. 
Author(s)

Byeongdu Lee, Chieh-Tsung Lo, P. Thiyagarajan, Dong R. Lee, Zhongwei Niu, and Qian Wang 
Journal of

\section{Applied Crystallography}

ISSN 0021-8898

Received 8 July 2007

Accepted 17 October 2007

C 2008 International Union of Crystallography

Printed in Singapore - all rights reserved

\section{Structural characterization using the multiple scattering effects in grazing-incidence small-angle X-ray scattering}

\author{
Byeongdu Lee, ${ }^{\mathrm{a} *}$ Chieh-Tsung Lo, ${ }^{\mathrm{a}} \mathrm{P}$. Thiyagarajan, ${ }^{\mathrm{a}}$ Dong R. Lee, ${ }^{\mathrm{b}} \mathrm{Zhongwei} \mathrm{Niu}^{\mathrm{c}}$ \\ and Qian Wang ${ }^{\mathrm{c}}$
}

a Argonne National Laboratory, 9700 South Cass Avenue, Argonne, IL 60439, USA, ${ }^{\mathbf{b}}$ Pohang
Accelerator Laboratory, POSTECH, Pohang 790-784, Republic of Korea, and ${ }^{\mathbf{c} D e p a r t m e n t ~ o f ~}$
Chemistry and Biochemistry and the Nanocenter, University of South Carolina, 631 Sumter Street,
Columbia, SC 29208, USA. Correspondence e-mail: blee@aps.anl.gov

The multiple scattering effects present in grazing-incidence small-angle X-ray scattering (GISAXS) data and interference between them are addressed theoretically as well as experimentally with measurement of a series of patterns at different incident angles, referred to as 'incident-angle-resolved GISAXS' (IAR-GISAXS). X-ray reflectivity (XR), GISAXS and IAR-GISAXS of virus particles on Si-substrate supported-polystyrene films have been measured and all the data have been analyzed with appropriate formalisms. It was found that under certain conditions it is possible to extract the correct structural features of the materials from the GISAXS/IAR-GISAXS data using the kinematic SAXS formalisms, without the need to use the distorted-wave Born approximation. Furthermore, the Kiessig fringes in GISAXS enable the measurement of the average distance between the particle and the substrate, similar to the measurement of film thickness using the fringes in the XR data. It is believed that the methods developed here will expand the application of GISAXS as they enable the application of model-independent and kinematic SAXS theories to nanostructured two-dimensional ordered films.

\section{Introduction}

Several X-ray techniques in grazing-incidence geometry are available to study nanostructural features in thin films. X-ray reflectivity (XR) is a powerful technique to characterize the density variation in layered structures (Parratt, 1954) and grazing-incidence X-ray standing waves (GIXSW) for the determination of the positions of inorganic particles in a film, by measuring fluorescence signals from the particles (Bedzyk et al., 1989; Wang et al., 1991). While both techniques provide information on the particle position above the substrate, grazing-incidence small-angle X-ray scattering (GISAXS) allows the determination of the size and shape of particles either on (Roth et al., 2003; Renaud et al., 2003) or embedded (Stein et al., 2007; Busch et al., 2006; Narayanan et al., 2005; Lee, Yoon et al., 2005; Lee, Park et al., 2005; Babonneau et al., 1999) in thin films on solid substrates, as well as their average positions: the relative location of particles with respect to a film surface can be determined by comparing the intensities of GISAXS measured at incident angles below and above the critical angle of the film (Wang et al., 2007; Babonneau et al., 1999; Levine et al., 1989).

A key phenomenon that distinguishes the grazing-incidence scattering techniques from other transmission methods is the reflection whose intensity can be almost the same as that of the incident beam. Scattering from the reflected beam is called multiple scattering since it is scattered at least two times (Chang, 2004). In GISAXS, multiple scattering refers additionally to the interferences between the scattered radiation from the two strong beams: the reflected and incident beams. Thus, kinematic scattering theory that assumes no multiple scattering is no longer valid unless the reflectivity is negligible. Although the full dynamic scattering theory might be considered for the grazing-incidence technique, a relatively simpler theory such as the distorted-wave Born approximation (DWBA) has been successfully used, where the former does not limit how many times the incident photon is scattered, while the latter assumes that it undergoes a maximum of two scattering events: a reflection and a scattering by a nanostructure. The DWBA, first applied to grazing-incidence scattering by Vineyard (1982), followed by Sinha et al. (1988) for studies of the roughness of thin films, has become a standard theoretical tool to analyze the data from various grazingincidence techniques, such as XR (Sanyal et al., 1993), GIXSW (Narayanan et al., 2005; Lee et al., 2006), and GISAXS (Stein et al., 2007; Busch et al., 2006; Lee, Yoon et al., 2005; Lee, Park et al., 2005; Renaud et al., 2003; Lazzari, 2002; Rauscher et al., 1995).

Recently, we investigated the effect of interfacial interaction on the morphology of tobacco mosaic virus (TMV) using 
GISAXS (Lee, Lo et al., 2007). This study demonstrated that TMV deforms along its cross section, depending on the nature of its interaction with the substrate. Due to the presence of dominant multiple-scattering effects in the GISAXS data, we could not readily apply the SAXS form factor for a cylinder and hence had to derive a new formalism for a multi-shell cylinder using the DWBA. Modelling the GISAXS data required prior information on the overall size and shape of the particles of interest. In conventional SAXS, some information can been extracted by model-independent Guinier analysis for a dilute system, and Porod analyses (Guinier \& Fournet, 1955; Roe, 2000) developed under a kinematic approximation. Although GISAXS data does contain the structural information, it is not readily possible to extract correctly without the application of the DWBA. Thus the objectives of the present study are: (i) develop experimental methodology for GISAXS to maximize the information content, (ii) develop methods to derive structural features from GISAXS data by utilizing the classical SAXS formalism and avoid the need to use the DWBA, and (iii) provide a better description of GISAXS in terms of its similarities and differences when compared with other existing grazing-incidence techniques. Towards these goals, we measured XR, GISAXS and incident-angle-resolved GISAXS (IAR-GISAXS) (Lee, Park et al., 2007) on samples of TMV on a silicon-wafer supported-polystyrene (PS) film and carried out the analysis with the known formalisms for XR and GISAXS data. Based on this systematic investigation, we found new approaches for GISAXS analysis: we show that under certain criteria, discussed later, conventional SAXS formalisms can be applied to GISAXS data that reproduce the results from the DWBA analysis. We also demonstrate the usefulness of Kiessig fringes in the GISAXS data to determine the location of the particle, relative to the substrate. Finally, we compare the information content in GISAXS with other grazing-incidence techniques such as XR and GIXSW.

\section{Theory of GISAXS}

A schematic of the GISAXS setup and an atomic force microscopy (AFM) image of the sample are shown in Fig. 1. The scattering amplitude of a particle in the geometry based on the DWBA is given by (Lee, Yoon et al., 2005; Lazzari,
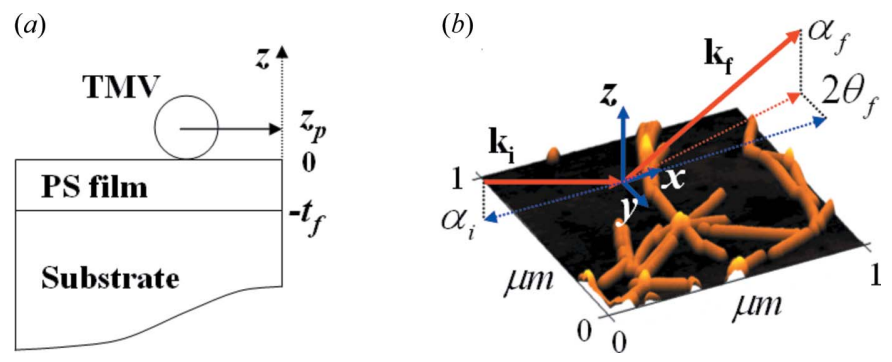

Figure 1

(a) Schematic of the structure of the samples. At the top surface of the PS film, $z=0$. (b) Definition of the geometry for GISAXS drawn on an AFM image of sample III.
2002; Rauscher et al., 1995; Sinha et al., 1988; Holy \& Baumbach, 1994)

$$
\begin{aligned}
\psi_{\mathrm{s}}^{l}\left(\alpha_{\mathrm{i}}, \alpha_{\mathrm{f}}, 2 \theta_{\mathrm{i}}, 2 \theta_{\mathrm{f}}\right)= & T_{\mathrm{i}}^{l} T_{\mathrm{f}}^{l} F\left(q_{\|}, q_{\mathrm{t}, z}^{l}\right)+T_{\mathrm{i}}^{l} R_{\mathrm{f}}^{l} F\left(q_{\|},-q_{\mathrm{r}, z}^{l}\right) \\
& +T_{\mathrm{f}}^{l} R_{\mathrm{i}}^{l} F\left(q_{\|}, q_{\mathrm{r}, z}^{l}\right)+R_{\mathrm{i}}^{l} R_{\mathrm{f}}^{l} F\left(q_{\|},-q_{\mathrm{t}, z}^{l}\right)
\end{aligned}
$$

and

$$
I \simeq\left|\sum_{l} \psi_{\mathrm{s}}^{l}\right|^{2}
$$

Here $\alpha_{\mathrm{i}}$ and $\alpha_{\mathrm{f}}$ are the incident and the exit angles in the plane of incidence and $2 \theta_{\mathrm{i}}$ and $2 \theta_{\mathrm{f}}$ are the incident and the exit angles along the normal to the plane of incidence, respectively. Superscript $l=0,1,2$, denote vacuum, polymer film and substrate in the experiment, and $T_{\mathrm{i}}^{l}$ and $R_{\mathrm{i}}^{l}$ are the amplitudes of the transmitted and reflected electric fields of an incoming wave in the $l$ th layer, respectively. For $l=0, T_{\mathrm{i}}=1$ and $T_{\mathrm{f}}=1$. The amplitude of particle scattering $F=$ $F_{0}\left(q_{\|}, q_{n, z}^{l}\right) \exp \left(-i q_{n, z}^{l} z_{\mathrm{p}}\right)$, where $F_{0}$ and $z_{\mathrm{p}}$ are the form factor and the $z$ position of a nanoparticle with respect to the top surface of the polymer film $(z=0)$, respectively. The form factor $F_{0}$ of the tobacco mosaic virus (TMV) with a cylindrical shape and with a length and diameter of about $300 \mathrm{~nm}$ and $18 \mathrm{~nm}$, respectively, will be discussed later. The subscripts $\mathrm{t}$ and $\mathrm{r}$ of the $q_{z}$ components denote the transmitted and reflected waves, respectively. The value of the in-plane scattering vector $q_{\|}=\left(q_{x}^{2}+q_{y}^{2}\right)^{1 / 2} \simeq q_{y}=k_{0} \cos \left(\alpha_{\mathrm{f}}\right) \sin \left(2 \theta_{\mathrm{f}}\right), k_{0}=$ $2 \pi / \lambda$, where $\lambda$ is the wavelength, as $2 \theta_{\mathrm{i}}=0$ due to the fact that the TMVs are randomly oriented in the in-plane direction. There are two out-of-plane scattering vectors in GISAXS: $q_{\mathrm{t}, z}^{l}=k_{\mathrm{f}, z}^{l}-k_{\mathrm{i}, z}^{l}$ and $q_{\mathrm{r}, z}^{l}=k_{\mathrm{f}, z}^{l}+k_{\mathrm{i}, z}^{l}$, where $k_{i, z}^{l}$ and $k_{\mathrm{f}, z}^{l}$ are outof-plane components of the incident and exit wavevectors at the $l$ th layer, respectively. Here we consider a dilute system wherein interparticle interactions are minimal. In all samples, the TMVs are considered to lie on a polymer film and equations (1) and (2) are used to fit the GISAXS data. When not specified in the equations, it is assumed that $l=0$ (vacuum).

\section{Experimental}

\subsection{Sample preparation}

A $10 \mathrm{mg} \mathrm{ml}^{-1}$ stock solution of TMV (U1 strain, from Dr Vicki Vance of the University of South Carolina) in $0.01 \mathrm{M}$ potassium phosphate buffer was diluted to $0.2 \mathrm{mg} \mathrm{ml}^{-1}$ with deionized water. An equal volume of methanol was added to the TMV solution and the solution was spun-cast on Si-wafer supported-PS films, which were prepared with thicknesses of 24.8, 45.0 and $60.3 \mathrm{~nm}$, designated as samples I, II and III, respectively. In all these samples, PS serves as a spacer to position the TMV at different distances above the solid substrate.

\subsection{GISAXS measurements}

GISAXS measurements were carried out at Sectors 8 and 12 at the Advanced Photon Source of Argonne National Laboratory. At sectors 8 and 12 we used $7.38 \mathrm{keV} \mathrm{X-rays}$ 
collimated to a beam size of $100 \times 50 \mu \mathrm{m}$ on a sample mounted in a vacuum chamber. The critical angles of PS and $\mathrm{Si}$ wafer for the above X-ray energy are $0.17^{\circ}$ and $0.24^{\circ}$, respectively. Scattered photons were detected using an area detector (MarCCD165) at about $2 \mathrm{~m}$ downstream from the sample. Source divergence and energy resolution of both beamlines are around $7 \mu \mathrm{rad}$ and $10^{-4}$, respectively.

\section{Results and discussion}

\subsection{The form factor of TMV on a substrate}

Fig. 2 shows the representative GISAXS images of sample III measured at the incident angles of $0.12,0.18,0.25$ and $0.4^{\circ}$. Since the TMV distribution on a Si-wafer supported-polymer film is dilute in all cases, the GISAXS pattern shows only their form-factor scattering. The form factor of a long cylinder can be described as a function of its length and diameter of cross section. Due to random orientation of the cylindrical TMVs on the film, their length exhibits a $q_{\|}^{-1}$ power-law scattering in the in-plane direction along $2 \theta_{\mathrm{f}}$, while the circular cross section produces concentric intensity lobes on the twodimensional detector plane around the direct beam position: M1 and M2 in Fig. 2(a) denote the first and second minima of the intensity lobes, respectively. Although the concentric lobes due to the circular cross section of TMV are readily seen in the data measured at an incident angle $\alpha_{\mathrm{i}}$ below the critical angle of the substrate, $\alpha_{\mathrm{c}, \mathrm{s}}$ in Figs. 2( $a$ ) and 2(b), they are barely seen in Figs. 2(c) and 2(d) measured at $\alpha_{\mathrm{i}}>\alpha_{\mathrm{c}, \mathrm{s}}$. This difference is caused by the reflectivity of the substrate as a function of $\alpha_{\mathrm{i}}$ : For all patterns shown in Fig. 2, the Guinier region of
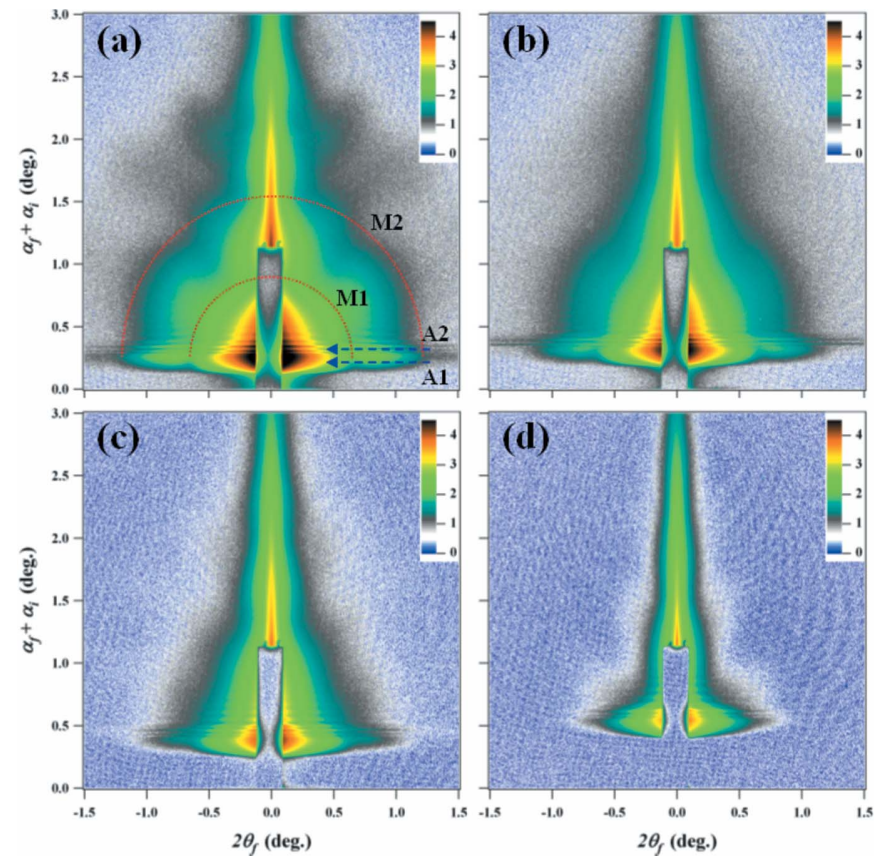

Figure 2

Representative GISAXS images measured at incident angles of $(a) 0.12$, (b) 0.18 , (c) 0.25 and $(d) 0.4^{\circ}$ for sample III. A1, A2, M1 and M2 in (a) denote the critical angles of PS film, Si substrate, and first and second minima of a scattering lobe, respectively. the scattering by the incident beam, with coordinates $\left(2 \theta_{\mathrm{f}}, \alpha_{\mathrm{f}}+\alpha_{\mathrm{i}}\right)=(0,0)$, is blocked by the substrate tilted for the incident angle, and thus it is not possible to determine the size of particles from the patterns. It should be noted that at an incident angle $\alpha_{\mathrm{i}}$ below the critical angle $\alpha_{\mathrm{c}, \mathrm{s}}$ where $R_{\mathrm{i}} \simeq 1$, the intensity of the reflected beam that bounces off the substrate will be the same as that of the incident beam. Thus the strong reflected beam serves as an additional direct beam, generating another set of scattering rays around the specularly reflected beam position $\left[\left(2 \theta_{\mathrm{f}}, \alpha_{\mathrm{f}}+\alpha_{\mathrm{i}}\right)=\left(0,2 \alpha_{\mathrm{i}}\right)\right]$. As the incident angle increases, the intensity of the reflected beam decreases proportionally to the reflectivity of the substrate, and the footprint of the X-ray beam becomes smaller, resulting in weaker scattering signals from particles distributed twodimensionally on substrates (Wang et al., 2007). Thus, the GISAXS data measured at $\alpha_{\mathrm{i}}<\alpha_{\mathrm{c}, \mathrm{s}}$ can provide information on the form factor of TMV. Although equation (1), derived with the DWBA, is typically used to analyze the GISAXS data, here we present a new approximation that enables the use of conventional SAXS formulae to analyze the data.

\subsection{Single-beam approximation (SBA)}

While the dynamic theory, equations (1) and (2), offers the best solution to fit the GISAXS images measured at $\alpha_{\mathrm{i}}<\alpha_{\mathrm{c}, \mathrm{s}}$, developing a kinematic approximation for GISAXS will enable application of the well known Guinier and Porod analyses, and thus extend its application. For instance, recently GISAXS has been shown to be useful to determine phases of the diffraction peaks (Lee, Park et al., 2007) and X-ray imaging (Vartanyants et al., 2007) using approximations similar to those proposed here. We demonstrate that at $\alpha_{\mathrm{i}}<\alpha_{\mathrm{c}, \mathrm{f}}$ (critical angle of the film), we can ignore the existence of two direct beams and assume the scattering to emanate from a single beam, which we refer to as the single-beam approximation (SBA). We simplified equation (1) as follows:

$$
\begin{aligned}
& I\left(\alpha_{\mathrm{i}}, \alpha_{\mathrm{f}}\right) \simeq\left|T_{\mathrm{i}}^{l}\right|^{2}\left|T_{\mathrm{f}}^{l}\right|^{2} \mid F_{0}\left(\mathbf{q}_{\mathrm{t}}^{l}\right) \exp \left(-i q_{\mathrm{t}, z}^{l} z_{\mathrm{p}}\right) \\
& \\
& +\left.B_{\mathrm{i}} F_{0}\left(\mathbf{q}_{\mathrm{r}}^{l}\right) \exp \left(-i q_{\mathrm{r}, z}^{l} z_{\mathrm{p}}\right)\right|^{2} \\
& =\left|T_{\mathrm{i}}^{l}\right|^{2}\left|T_{\mathrm{f}}^{l}\right|^{2}\left[\left|F_{0}\left(\mathbf{q}_{\mathrm{t}}^{l}\right)\right|^{2}+\left|B_{\mathrm{i}}^{l}\right|^{2}\left|F_{0}\left(\mathbf{q}_{\mathrm{r}}^{l}\right)\right|^{2}+D\right] \\
& \quad \text { for } \begin{array}{l}
\alpha_{\mathrm{i}}<\alpha_{\mathrm{c}, \mathrm{s}}, \\
\alpha_{\mathrm{f}}>\alpha_{\mathrm{c}, \mathrm{s}},
\end{array} \\
& I\left(\alpha_{\mathrm{i}}, \alpha_{\mathrm{f}}, l=0\right)=\left|F_{0}\left(\mathbf{q}_{\mathrm{f}}\right)\right|^{2}+\left|F_{0}\left(\mathbf{q}_{\mathrm{r}}\right)\right|^{2}+D \text { for } \begin{array}{l}
\alpha_{\mathrm{i}}<\alpha_{\mathrm{c}, \mathrm{f}}, \\
\alpha_{\mathrm{f}}>\alpha_{\mathrm{c}, \mathrm{s}},
\end{array}
\end{aligned}
$$

where $D=2 F_{0}\left(\mathbf{q}_{\mathrm{t}}^{l}\right) F_{0}\left(\mathbf{q}_{\mathrm{r}}^{l}\right)|B| \cos (\delta+v), B_{\mathrm{i}}=R_{\mathrm{i}} / T_{\mathrm{i}}, \mathbf{q}_{\mathrm{t}}=\left(q_{\|}\right.$, $\left.q_{\mathrm{t}, z}\right)$, and $\mathbf{q}_{\mathrm{r}}=\left(q_{\|}, q_{\mathrm{r}, z}\right)$. $\delta$ is the phase angle of $B_{\mathrm{i}}, v$ is $\left(q_{\mathrm{t}, z}-q_{\mathrm{r}, z}\right) z_{\mathrm{p}}$, and $\Delta q_{z} \equiv\left(q_{\mathrm{t}, z}-q_{\mathrm{r}, z}\right)=-2 k_{\mathrm{i}, z} \simeq 2 k_{0} \alpha_{\mathrm{i}}$. As shown in Fig. 3(a), the only difference between the calculations with the full equation and equation $(3 a)$ is the presence of fringes in a wave-guiding region (WGR), at $\alpha_{\mathrm{c}, \mathrm{f}}<\alpha_{\mathrm{f}}<\alpha_{\mathrm{c}, \mathrm{s}}$. The origin of these fringes will be discussed in the next section. Equation (3a) and Fig. 3(a) suggest that four terms that were in equation (1) can be reduced to two, which limits the observation of features around the WGR. But the limitation 

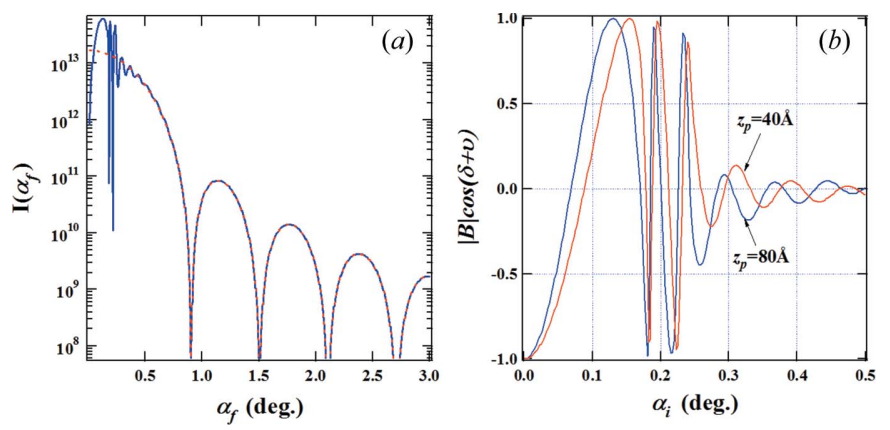

Figure 3

(a) Full DWBA calculation (blue solid line) and the approximation of equation ( $3 a$ ) for a sphere of radius $R=80 \AA . \alpha_{\mathrm{i}}=0.131^{\circ} \cdot 2 \theta_{\mathrm{f}}=0^{\circ}$. Film thickness $=500 \AA$. X-ray wavelength $=1.68 \AA . \quad z_{\mathrm{p}}=R / 2$. Electron densities of the polymer film and $\mathrm{Si}$ substrate are $0.338 \AA^{-1}$ and $0.699 \AA^{-1}$ and thus $\alpha_{\mathrm{c}, \mathrm{f}}$ and $\alpha_{\mathrm{c}, \mathrm{s}}$ are $0.17^{\circ}$ and $0.24^{\circ}$, respectively. $(b)$ $|B| \cos (\delta+v)$ as a function of $\alpha_{\mathrm{i}}$ for spheres of radius $R=40$ and $80 \AA$. The other parameters are the same as those in $(a)$.

could be overcome by applying the standing-wave concept (see $\$ 4.5)$.

When we consider only the particles on the top surface of a substrate $(l=0)$, equation ( $3 a)$ reduces to equation $(3 b)$ and $T_{\mathrm{i}}$ and $T_{\mathrm{f}}$ will become 1 . Furthermore, for specular reflectivity $\left|B_{\mathrm{i}}\right|^{2}=1$ at $\alpha_{\mathrm{i}}<\alpha_{\mathrm{c}, \mathrm{f}}$, and will tend to 1 at $\alpha_{\mathrm{i}}<\alpha_{\mathrm{c}, \mathrm{s}}$ when the contribution from the film (very thin or composed of low- $Z$ materials) to the reflectivity is small in the above angular range. The contribution of $D$ is determined by the value of $|B| \cos (\delta+v)$, which for particles located at $z_{\mathrm{p}}=40$ and $80 \AA$ is plotted in Fig. 3(b) as a function of $\alpha_{\mathrm{i}}$. Note that its frequency is higher at larger $z_{\mathrm{p}}$. Thus, overall GISAXS intensity will become maximum and minimum at $|B| \cos (\delta+v)=1$ and -1 , respectively, when the sign of $F_{0}\left(\mathbf{q}_{\mathrm{t}}\right)$ and $F_{0}\left(\mathbf{q}_{\mathrm{r}}\right)$ are the same. This suggests that modulation of GISAXS intensity will occur as a function of $\alpha_{\mathrm{i}}$ following the variation of $D$, which is determined mostly by $z_{\mathrm{p}}$ and $t_{\mathrm{f}}$ (film thickness). Note that $I=$ $\left|F_{0}\left(\mathbf{q}_{\mathrm{t}}\right)+F_{0}\left(\mathbf{q}_{\mathrm{r}}\right)\right|^{2}$ for $D=1, I=\left|F_{0}\left(\mathbf{q}_{\mathrm{t}}\right)-F_{0}\left(\mathbf{q}_{\mathrm{r}}\right)\right|^{2}$ for $D=-1$, and $I=\left|F_{0}\left(\mathbf{q}_{\mathrm{t}}\right)\right|^{2}+\left|F_{0}\left(\mathbf{q}_{\mathrm{r}}\right)\right|^{2}$ for $D=0$. In addition, equations $3(a)$ and $3(b)$ suggest that $D$ will be cancelled due to the destructive interference if particles are randomly distributed in three dimensions, or with random $z_{\mathrm{p}}$ values.

Even more generally, as long as the particle size is less than a certain value, $D$ can be neglected, leading to a further simplification of equation $3(b)$ as follows. For a sphere of radius $R$, the small-size-particle approximation refers to the conditions such that $\Delta q_{z}$ is less than about a quarter of the period of $F_{0}\left(q_{\|}, q_{z}\right)$ along $q_{z}$ so that the first minima of $F_{0}$ appear at $q_{z}>\Delta q_{z}$, or more simply $\Delta q_{z}$ will fall within a Guinier region $\left.\left(q_{z}\right)<1 / R\right)$. When the small-sizeparticle approximation is applied, $F_{0}\left(\mathbf{q}_{\mathrm{r}}\right) \simeq F_{0}\left(\mathbf{q}_{\mathrm{t}}\right)$ and $2 F_{0}\left(\mathbf{q}_{\mathrm{t}}\right) F_{0}\left(\mathbf{q}_{\mathrm{r}}\right) \simeq\left|F_{0}\left(\mathbf{q}_{\mathrm{t}}\right)\right|^{2}+\left|F_{0}\left(\mathbf{q}_{\mathrm{r}}\right)\right|^{2}$. Then, GISAXS intensity will be proportional to $\left[\left|F_{0}\left(\mathbf{q}_{\mathrm{t}}\right)\right|^{2}+\left|F_{0}\left(\mathbf{q}_{\mathrm{r}}\right)\right|^{2}\right]$ with a scale factor of $1-|B| \cos (\delta+v)$. For a sphere, the first minimum appears at $q=4.5 / R$, which leads to the requirement that $\alpha_{\mathrm{i}} \ll 4.5 /\left(2 k_{0} R\right)$ or $\alpha_{\mathrm{i}}<1 /\left(2 k_{0} R\right)$.

Note that $q_{\mathrm{t}, z}=k_{\mathrm{f}, z}+\Delta q_{z} / 2$ and $q_{\mathrm{r}, z}=k_{\mathrm{f}, z}-\Delta q_{z} / 2$. Thus, $\left[\left|F_{0}\left(\mathbf{q}_{\mathrm{t}}\right)\right|^{2}+\left|F_{0}\left(\mathbf{q}_{\mathrm{r}}\right)\right|^{2}\right] \simeq\left[\left|F_{0}\left(q_{\|}, k_{\mathrm{f}, z}+\Delta q_{z} / 2\right)\right|^{2}+\right.$ $\left.\left|F_{0}\left(q_{\|}, k_{\mathrm{f}, z}-\Delta q_{z} / 2\right)\right|^{2}\right]$. According to the Guinier approx- imation $\left[I(q) \simeq \exp \left(-R_{\mathrm{g}}^{2} q^{2} / 3\right) \simeq 1-R_{\mathrm{g}}^{2} q^{2} / 3\right]$, it would become $2-R_{\mathrm{g}}^{2}\left(2 q_{\|}^{2}+q_{\mathrm{t}, z}^{2}+q_{\mathrm{r}, z}^{2}\right) / 3$, where $R_{\mathrm{g}}$ is the radius of gyration and $q_{\mathrm{t}, z}^{2}+q_{\mathrm{r}, z}^{2}=2\left(k_{\mathrm{f}, z}^{2}+\Delta q_{z}^{2} / 4\right)$. Thus, $I \simeq 2-2 R_{\mathrm{g}}^{2}\left(q_{\|}^{2}+k_{\mathrm{f}, z}^{2}+\right.$ $\left.\Delta q_{z}^{2} / 4\right) / 3 \simeq 2\left|F\left[q_{\|},\left(k_{\mathrm{f}, z}^{2}+\Delta q_{z}^{2} / 4\right)^{1 / 2}\right]\right|^{2}$. When $k_{\mathrm{f}, z}^{2} \gg \Delta q_{z}^{2} / 4$, or $2 \alpha_{\mathrm{f}} \gg \alpha_{\mathrm{i}}$, it finally becomes

$$
I\left(\alpha_{\mathrm{f}}, l=0\right) \simeq 2\left|F_{0}\left(q_{\|}, k_{\mathrm{f}, z}\right)\right|^{2}, \text { for } \begin{aligned}
& \alpha_{\mathrm{i}}<\alpha_{\mathrm{c}, \mathrm{f}}, \\
& \\
& \Delta q_{z} R \ll 4.5 .
\end{aligned}
$$

This suggests that the sum of the two scatterings components $\left[\left|F_{0}\left(\mathbf{q}_{\mathrm{t}}\right)\right|^{2}+\left|F_{0}\left(\mathbf{q}_{\mathrm{r}}\right)\right|^{2}\right]$ defined by two respective scattering vectors $\mathbf{q}_{\mathrm{t}}$ and $\mathbf{q}_{\mathrm{r}}$ can be approximated to a single term $\left[2\left|F_{0}\left(\mathbf{q}_{\mathrm{i}}\right)\right|^{2}\right]$ defined by a new scattering vector $\mathbf{q}_{\mathrm{i}} \equiv\left[q_{\|}\right.$, $\left.\left(k_{\mathrm{f}, z}^{2}+\Delta q_{z}^{2} / 4\right)^{1 / 2}\right] \simeq\left(q_{\|}, k_{0} \alpha_{\mathrm{f}}\right)$ (note that $\left.k_{\mathrm{f}, z} \simeq k_{0} \alpha_{\mathrm{f}}\right)$ where subscript $\mathrm{i}$ denotes the 'imaginary single beam'. In other words, as long as $\alpha_{\mathrm{i}}<\alpha_{\mathrm{c}, \mathrm{f}}$, GISAXS by two incident beams can be approximated to SAXS by a single beam, called the 'imaginary single beam'.

In Fig. 4, we compare the curves $I_{\mathrm{i}}$ calculated with the SBA, as well as $I_{\mathrm{DWBA}}$ calculated with the DWBA, for the case of $R=$ 40 and $80 \AA$. All curves are normalized by the maximum intensities for a better comparison of form factors. For both sizes, as discussed above, locations of maxima and minima of $I_{\text {DWBA }}$ curves calculated for different $\alpha_{\mathrm{i}}$ values are identical as long as $\alpha_{\mathrm{i}}<\alpha_{\mathrm{c}, \mathrm{f}}$ (see Figs. $4 a$ and $4 b$ ) and are well reproduced by $I_{\mathrm{i}}$. However, the deviation between $I_{\mathrm{DWBA}}$ and $I_{\mathrm{i}}$ increases with increasing $\alpha_{\mathrm{i}}$ as well as the particle size (see Figs. $4 c$ and $4 d)$. Furthermore, at $\alpha_{\mathrm{i}}$ values of approximately 0.18 and $0.22^{\circ}$ for a sphere of radius $R=80 \AA$ (see Fig. $3 b$ ) where

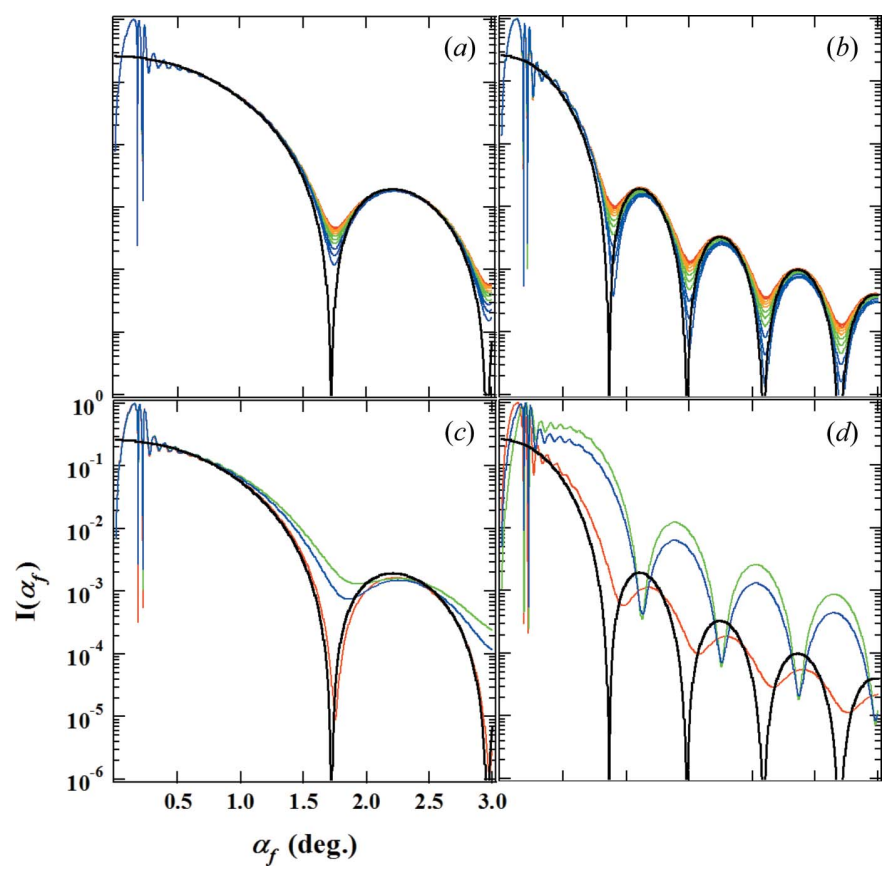

Figure 4

Simulations of GISAXS curves for spheres of radius $R=40 \AA[(a)$ and $(c)]$ and $80 \AA[(b)$ and $(d)]$. Incident angles for $(a)$ and $(b)$ are 0.01 to $0.12^{\circ}$ with $0.01^{\circ}$ step (in red, orange, green and blue, from low angle to high angle), and for $(c)$ and $(d)$ are $0.16^{\circ}$ (red), $0.18^{\circ}$ (green) and $0.21^{\circ}$ (blue). Intensities calculated with SBA are shown in thick black curves. See the caption to Fig. 3(a) for other parameters. 
$|B| \cos (\delta+v)=-1$, the maxima of the form factor appear at the position of its minima as per the SBA. A similar feature was found for a smaller sphere of radius $R=40 \AA$ at $\alpha_{\mathrm{i}}=0.185$ and $0.225^{\circ}$ (not shown). This is because, at this condition $I \simeq$ $\left|F_{0}\left(\mathbf{q}_{\mathrm{t}}\right)-F_{0}\left(\mathbf{q}_{\mathrm{r}}\right)\right|^{2}$.

The intrinsic limitation of GISAXS to obtain correct size distributions is as follows. As can be seen from equation 3(b), GISAXS patterns are smeared by two beams, and thus the depth of the minima of the form factor highly depends on $D$ (or $\alpha_{\mathrm{i}}$ ) at $\alpha_{\mathrm{i}}<\alpha_{\mathrm{c}, \mathrm{s}}$, which might affect the delineation of accurate size distribution. In the range of $\alpha_{\mathrm{i}}<\alpha_{\mathrm{c}, \mathrm{f}}$, the minima of the form factor become deeper as $|B| \cos (\delta+v)$ approaches to 1 (see Fig. $3 b$ ), suggesting that GISAXS has to be measured at the $\alpha_{\mathrm{i}}$ satisfying $|B| \cos (\delta+v)=1$ to obtain the deepest minima to maximize the resolution for the measurement of size distribution. It might be useful to make measurements at least at two $\alpha_{\mathrm{i}}$ values for better accuracy in polydispersity.

The measured GISAXS patterns for the TMV with cross section of about $16 \mathrm{~nm}$ diameter clearly exhibit the features discussed for the SBA: curves measured at smaller incident angles nicely overlap in the $I$ versus $\alpha_{\mathrm{f}}$ plot, showing identical positions of minima and maxima (see Fig. $5 a$ ). The minima progressively become shallow with increasing $\alpha_{\mathrm{i}}$. The phase swapping, wherein the maxima appear at the positions of the minima, can also be clearly identified at the $\alpha_{\mathrm{i}}$ where the overall GISAXS intensities are lower than those measured at the other angles (see Fig. 11) because $I \simeq\left|F_{0}\left(\mathbf{q}_{\mathrm{t}}\right)-F_{0}\left(\mathbf{q}_{\mathrm{r}}\right)\right|^{2}$ at the angle, which corresponds to the minima of $|B| \cos (\delta+v)$. The intensity modulation as a function of $\alpha_{\mathrm{i}}$ caused by $|B| \cos (\delta+v)$ is termed the Kiessig fringes along the $\alpha_{\mathrm{i}}$ direction (see the next section). This can be used for determining or refining the phase of the form factor $F_{0}(\mathbf{q})$ (Lee, Park et al., 2007).

\subsection{The Kiessig fringes}

In addition to the concentric modulation of the form factor of TMV, finer modulation lines parallel to the $2 \theta_{\mathrm{f}}$ axis are also observed in the GISAXS pattern in Fig. 2. The latter modulations are more distinct in a wave-guiding region, at $\alpha_{\mathrm{c}, \mathrm{f}}<\alpha_{\mathrm{f}}<\alpha_{\mathrm{c}, \mathrm{s}}$ (see A1 and A2 in Figs. 2 and 5a). As shown in Fig. 5(a), the fringes persist even at $\alpha_{\mathrm{f}}>\alpha_{\mathrm{c}, \mathrm{s}}$ and almost
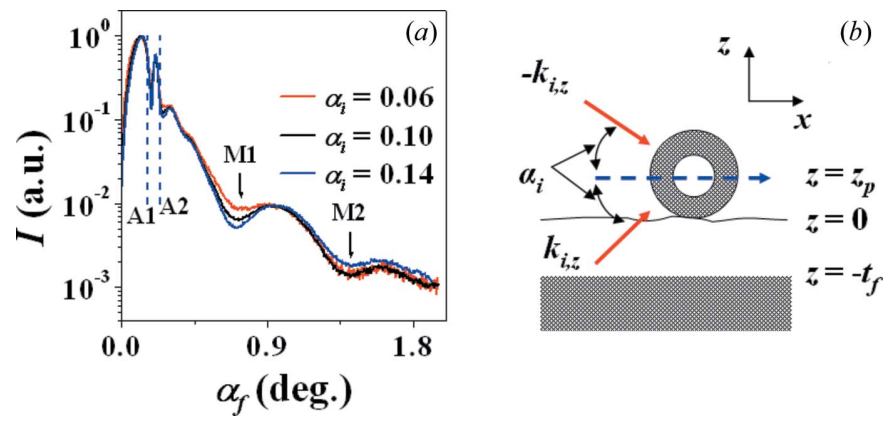

Figure 5

(a) Vertical cuts of GISAXS images measured at three incident angles for sample I. Cuts were made at $2 \theta_{\mathrm{f}}=0.15^{\circ}$. (b) The imaginary single incident beam is shown using a dotted arrow. disappear at an angle close to $0.45^{\circ}$. Similar to the minima (M1 and M2) of the form factor, these fringes are also incidentangle independent for the curves measured at $\alpha_{\mathrm{i}}<\alpha_{\mathrm{c}, \mathrm{f}}$ : their periods and shapes overlap exactly when plotted as a function of the exit angle $\left(\alpha_{\mathrm{f}}\right)$, which indicate that the periods of these fringes are a function of $\alpha_{\mathrm{f}}$ in the incident-angle ranges.

The evidence for the origin of these fringes can be seen in equation (1). The region where the fringes appear is close to the Guinier region, and thus we can use the small-particle approximation and $F_{0} \simeq 1$. The equation then becomes

$$
\begin{aligned}
I\left(\alpha_{\mathrm{i}}, \alpha_{\mathrm{f}}\right)= & \mid\left[T_{\mathrm{i}} \exp \left(i k_{\mathrm{i}} z_{\mathrm{p}}\right)+R_{\mathrm{i}} \exp \left(-i k_{\mathrm{i}} z_{\mathrm{p}}\right)\right] \\
& \times\left.\left[T_{\mathrm{f}} \exp \left(-i k_{\mathrm{f}} z_{\mathrm{p}}\right)+R_{\mathrm{f}} \exp \left(i k_{\mathrm{f}} z_{\mathrm{p}}\right)\right]\right|^{2} \\
= & \left|\psi_{\mathrm{i}}\left(\alpha_{\mathrm{i}}\right) \psi_{\mathrm{f}}\left(\alpha_{\mathrm{f}}\right)\right|^{2} \\
= & \left|\psi_{\mathrm{i}}\left(\alpha_{\mathrm{i}}\right)\right|^{2}\left|\psi_{\mathrm{f}}\left(\alpha_{\mathrm{f}}\right)\right|^{2} .
\end{aligned}
$$

The electric fields $\psi_{\mathrm{i}}$ and $\psi_{\mathrm{f}}$ are termed as the X-ray standing waves because their nodes sit at the interface of the film and substrate (Lee et al., 2006). Note that the fringes in GISAXS measured at a given $\alpha_{\mathrm{i}}$ are from $\psi_{\mathrm{f}}$ rather than $\psi_{\mathrm{i}}$ as the latter only acts as a coefficient that determines the intensity scale of the two-dimensional pattern at a given $\alpha_{\mathrm{i}}$. Furthermore, when a particle is located at a node of $\psi_{\mathrm{i}}\left(\alpha_{\mathrm{i}}\right)$, the scattering intensity from the particle will become the lowest (Narayanan et al., 2005). On the other hand, when the particle sits at the antinode, it will produce the highest scattering intensity. The standing-wave concept seems to be a good explanation for the origin of the fringes.

When the size of the particle is large, the fringes become incident-angle dependent at $\alpha_{\mathrm{i}}>\alpha_{\mathrm{c}, \mathrm{f}}$ because values of $F\left(\mathbf{q}_{\mathrm{t}}\right)$ and $F\left(\mathbf{q}_{\mathrm{r}}\right)$ in equation (1) are not equal to each other and thus the approximation in equation (4) is no longer valid and the standing-wave concept is not appropriate to explain the origin of fringes because $F(\mathbf{q})$ will start to interfere with $\psi_{\mathrm{i}}$ or $\psi_{\mathrm{f}}$. The incident-angle dependence of the fringes is shown in Fig. 6: the fringes for the top two curves measured at $\alpha_{\mathrm{i}}<\alpha_{\mathrm{c}, \mathrm{f}}\left(\alpha_{\mathrm{c}, \mathrm{f}} \simeq\right.$ $0.17^{\circ}$ ) resemble each other, as shown in Fig. 5(a) already. But,

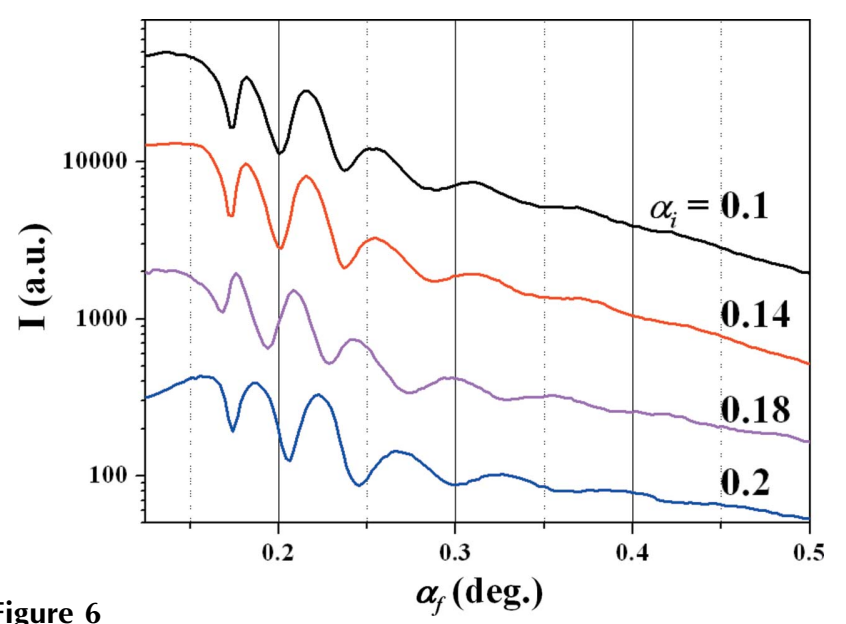

Vertical cuts of GISAXS images measured at four different incident angles for sample III. Cuts were made at $2 \theta_{\mathrm{f}}=0.15^{\circ}$. For the top two curves, $\alpha_{\mathrm{i}}<\alpha_{\mathrm{c}, \mathrm{f}}$, and for the others, $\alpha_{\mathrm{c}, \mathrm{f}}<\alpha_{\mathrm{i}}<\alpha_{\mathrm{c}, \mathrm{s}}$. 
those at $\alpha_{\mathrm{i}}>\alpha_{\mathrm{c}, \mathrm{f}}$ show that the maxima and minima of the fringes appear at different locations, which is not expected for very small particles. As equation (1) already implies, the fringes are the result of the interference between the scattered waves generated by the two incident beams. Note that the period of the fringes does not change in Fig. 6 regardless of the incident angles, from which the distance between the center of the particle to the interface of film/substrate can be calculated (see §4.5). Based on the above discussion, we refer to the fringes as the Kiessig fringes (Kiessig, 1931) at off-specular condition (Kapp \& Wainfan, 1965). It is interesting to note that the period of the intensity modulation as a function of $\alpha_{\mathrm{i}}$, caused by $\left|B_{\mathrm{i}}\right| \cos (\delta+v)$, is the same as that of $\psi_{\mathrm{i}}$. Since the fringes in the two-dimensional images in Figs. 2, 5(a) and 6 appear along the $\alpha_{\mathrm{f}}$ axis, they will be called the Kiessig fringes on the $\alpha_{\mathrm{f}}$ side, and similarly the intensity modulation as a function of $\alpha_{\mathrm{i}}$ can be called the Kiessig fringes on the $\alpha_{\mathrm{i}}$ side.

The results derived in $\$ \$ 4.2$ and 4.3 suggest that a good approximation of GISAXS measured at $\alpha_{\mathrm{i}}<\alpha_{\mathrm{c}, \mathrm{f}}$ is

$$
I\left(\alpha_{\mathrm{i}}, \alpha_{\mathrm{f}}\right)=\left|\psi_{\mathrm{i}}\left(\alpha_{\mathrm{i}}\right)\right|^{2}\left|\psi_{\mathrm{f}}\left(\alpha_{\mathrm{f}}\right)\right|^{2}\left|F_{0}\left(\mathbf{q}_{\mathrm{i}}\right)\right|^{2}, \quad \alpha_{\mathrm{i}} \leq \alpha_{\mathrm{c}, \mathrm{f}} .
$$

Here, the GISAXS measured at $\alpha_{\mathrm{i}} \leq \alpha_{\mathrm{c}, \mathrm{f}}$ seems like the pattern generated by the SBA parallel to the surface of a substrate.

\subsection{IAR-GISAXS}

We measured GISAXS data at $\alpha_{\mathrm{i}}$ from 0.01 to $0.45^{\circ}$ in $0.005^{\circ}$ steps and sliced the images at $2 \theta_{\mathrm{f}}=0.15^{\circ}$ to produce vertical cuts with intensity versus $\alpha_{\mathrm{i}}+\alpha_{\mathrm{f}}$. The vertical cuts taken from the data at various incident angles are stacked to form an IAR-GISAXS image as shown in Fig. 7, where the abscissa is $\alpha_{\mathrm{i}}$ and the ordinate is $\alpha_{\mathrm{i}}+\alpha_{\mathrm{f}}$. This IAR-GISAXS image clearly shows how the scattering profiles change as a function of $\alpha_{\mathrm{i}}$, and the key components (the Kiessig fringes, form factor and critical angles) that are necessary to determine the structure and position of TMV are distinctly seen. The first and second minima of scattering lobes are designated as $\mathrm{M} 1$ and $\mathrm{M} 2$ in Fig. 7, respectively, and $\alpha_{\mathrm{c}, \mathrm{f}}$ and $\alpha_{\mathrm{c}, \mathrm{s}}$ are shown as A1 and A2, respectively. The angle of the sample $\left[\alpha_{\mathrm{f}}=0\right]$ can also be clearly distinguished because there is no intensity below the $\alpha_{\mathrm{f}}=0$ line. As discussed earlier in $\S 4.2$, the GISAXS profile is a function of $k_{0} \alpha_{\mathrm{f}}$ at $\alpha_{\mathrm{i}}<\alpha_{\mathrm{c}, \mathrm{f}}$, which is clearly proved by traces denoted by M1 and M2 that vary as a function of $\alpha_{\mathrm{i}}$ in Fig. 7. They move to higher $\alpha_{\mathrm{f}}$ that is proportional to $\alpha_{\mathrm{i}}$ at $\alpha_{\mathrm{i}}<\alpha_{\mathrm{c}, \mathrm{f}}$, and are parallel to A1 and A2. The traces of M1 and $\mathrm{M} 2$ can be exactly predicted by the SBA, where the position of the imaginary single beam is located at the $\alpha_{\mathrm{f}}=0$ line. They eventually become parallel to the $\alpha_{\mathrm{i}}$ axis, or independent of $\alpha_{\mathrm{i}}$ at $\alpha_{\mathrm{i}}>\alpha_{\mathrm{c}, \mathrm{s}}$, and deviate from the prediction based on the SBA, suggesting that the form-factor scattering is no longer a function of $\alpha_{\mathrm{i}}$ at $\alpha_{\mathrm{i}}>\alpha_{\mathrm{c}, \mathrm{s}}$ because $R_{\mathrm{i}} \simeq$ 0 and the reflected beam can no longer serve as a direct beam.

Interestingly, the $\alpha_{\mathrm{i}}=\alpha_{\mathrm{f}}$ line denoted as A3 in Fig. 7, is like a mirror symmetry line for the Kiessig fringes, both along the vertical $\left(\alpha_{\mathrm{i}}+\alpha_{\mathrm{f}}\right)$ axis and the horizontal $\left(\alpha_{\mathrm{i}}\right)$ axis. Since data on this line are measured symmetrically, i.e. $\alpha_{\mathrm{i}}=\alpha_{\mathrm{f}}$, we refer to

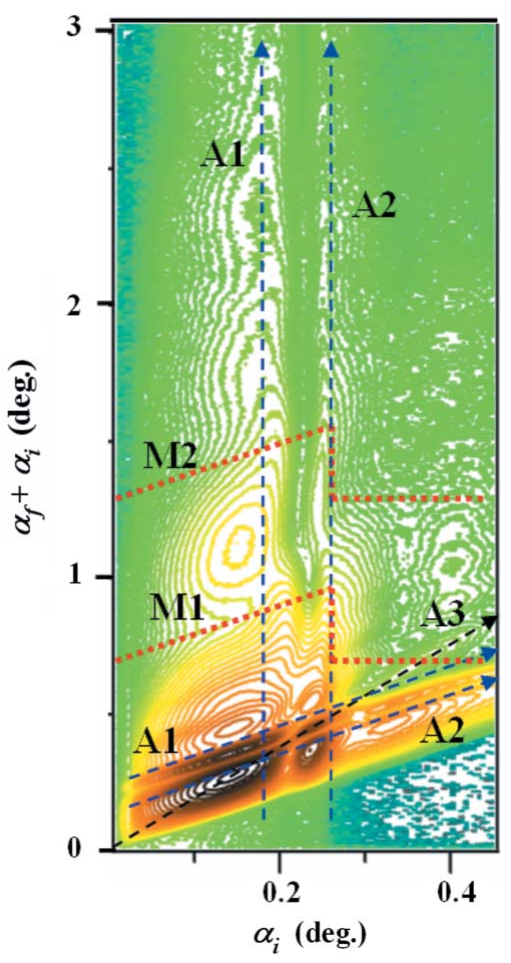

Figure 7

IAR-GISAXS image for sample I and vertical cuts of this image, made at $2 \theta_{\mathrm{f}}=0.15^{\circ}$. A1, A2 and A3 denote the angles $\alpha_{\mathrm{c}, \mathrm{f}}, \alpha_{\mathrm{c}, \mathrm{s}}$ and $\alpha_{\mathrm{i}}=\alpha_{\mathrm{f}}(\mathrm{SM}$ line), respectively. M1 and M2 are for the first and second minima of the form factor of the TMV, respectively. Note that A1 and A2 exist on both the horizontal and the vertical axis of the IAR-GISAXS image. The color scale is identical to that of Fig. 2.

this as the symmetric mode (SM). X-ray reflectivity is very similar to SM-GISAXS because both are measured symmetrically. The only difference between them is that the in-plane angle $2 \theta_{\mathrm{f}}$ is zero for XR and non-zero for SM-GISAXS. Based on this, the SM can be referred to as in-plane off-specular reflectivity.

\subsection{GISAXS versus XR}

In this section, we compare the Kiessig fringes of SMGISAXS and XR. Following the small-particle-size approximation, GISAXS intensity along the SM line in Fig. 7 is given by

$$
\begin{aligned}
I_{2 \theta_{\mathrm{f}} \neq 0} & =\left|\psi_{\mathrm{s}}\right|^{2} \\
& =\left|\psi_{\mathrm{i}}\right|^{2}\left|\psi_{\mathrm{f}}\right|^{2}\left|F_{0}(\mathbf{q})\right|^{2},
\end{aligned}
$$

where $T_{\mathrm{i}}=T_{\mathrm{f}}, R_{\mathrm{i}}=R_{\mathrm{f}}$, and $k_{\mathrm{f}, z}=-k_{\mathrm{i}, z} \cdot \psi_{\mathrm{i}}=\psi_{\mathrm{f}} \equiv T_{\mathrm{i}} \exp \left(i k_{\mathrm{i}, z} z_{\mathrm{p}}\right)$ $+R_{\mathrm{i}} \exp \left(-i k_{\mathrm{i}, z} z_{\mathrm{p}}\right)$. On the other hand, XR derived under the DWBA is expressed (Sanyal et al., 1993; Lee, Park et al., 2007) as

$$
I_{2 \theta_{\mathrm{r}}=0}=\left|r+\psi_{\mathrm{s}}\left(q_{z}\right)\right|^{2},
$$

where $r$ is the Fresnel reflectivity of the film on the substrate. When $r$ is much stronger than the scattering of particles, $\psi_{\mathrm{s}}\left(q_{z}\right)$, as in this work, equation (7) can be further simplified as 
Table 1

Fitting parameters.

Fitted by the Parratt formulae (Parratt, 1954). $\sigma_{\mathrm{s}}, t_{\mathrm{f}}, \sigma_{\mathrm{f}}, z_{\mathrm{p}}$ and $\sigma_{\mathrm{p}}$ are the roughness of a substrate, film thickness, film roughness, $z$ position of a particle from a surface of a film, and the distribution width of $z_{\mathrm{p}}$, respectively. $t_{\mathrm{f}, \mathrm{GISAXS}}$ is $t_{\mathrm{f}}$ determined by GISAXS.

\begin{tabular}{|c|c|c|c|c|c|c|c|}
\hline & \multicolumn{3}{|l|}{$\mathrm{XR}(\AA) \dagger$} & \multicolumn{4}{|c|}{ GISAXS $(\AA) \ddagger$} \\
\hline & $d_{\mathrm{XR}}$ & $\sigma_{\mathrm{s}}$ & $\sigma_{\mathrm{f}}$ & $d_{\text {GISAXS }} \S$ & $t_{\mathrm{f}, \mathrm{GISAXS}}$ & $z_{\mathrm{p}}$ & $\sigma_{\mathrm{p}}$ \\
\hline I & $248 \pm 1$ & 2 & $7 \pm 1$ & 328 & $296 \pm 1.8$ & $32 \pm 2$ & $40 \pm 10$ \\
\hline II & $450 \pm 4$ & 3 & $27 \pm 3$ & 530 & $481 \pm 3$ & $42 \pm 3$ & $49 \pm 10$ \\
\hline III & $603 \pm 3$ & 3 & $38 \pm 5$ & 689 & $641 \pm 3$ & $48 \pm 3$ & $50 \pm 10$ \\
\hline
\end{tabular}

† Electron densities $\left(\rho_{\mathrm{e}}\right)$ are 0.32 and $0.706 \AA^{-3}$, and absorption terms in the refractive index $(\beta)$ are $1.0 \times 10^{-9}$ and $2.2 \times 10^{-7}$ for the PS and the Si substrate, respectively. $\neq$ The radius $\left(R_{\mathrm{TMV}}\right)$ and its distribution width for a TMV particle are $73 \AA$ and $8 \AA$, respectively, as obtained from GISAXS. $\quad \S d_{\text {GISAXS }}=t_{\mathrm{f}, \text { GISAXS }}+z_{\mathrm{p}}$.

$$
I_{2 \theta_{\mathrm{f}}=0} \simeq|r|^{2}
$$

Here, we note the difference in the origin of the Kiessig fringes in GISAXS and XR. They are due to the amplitude $\psi_{\mathrm{f}}$ as a function of $t_{\mathrm{f}}+z_{\mathrm{p}}$ in GISAXS, and $r$ as a function of $t_{\mathrm{f}}$ in XR. When the scattering intensity $\psi_{\mathrm{s}}\left(q_{z}\right)$ is much stronger compared with $r$, as in the case of high-contrast systems, it will bring a greater contribution to XR and the scattering can be treated as originating from a layer with an average electron density.

We carried out simultaneous $\mathrm{XR}$ and IAR-GISAXS measurements and compared the XR data with the intensities in the SM-GISAXS data extracted at $\left(2 \theta_{\mathrm{f}}, \alpha_{\mathrm{f}}\right)=\left(0.15^{\circ}, \alpha_{\mathrm{i}}\right)(\mathrm{A} 3$ line in Fig. 7) and corrected for the length of the footprint of the X-ray beam (Wang et al., 2007). As expected, SM-GISAXS and XR show similar features in the overall curve shape, such as the presence of Kiessig fringes and reduced intensities above the critical angle of the substrate. However, notable differences have been found in the periods of the Kiessig fringes in the two curves: those in SM-GISAXS are shorter than those in XR for all the three samples, implying that the length scales generating oscillations in the GISAXS pattern are longer than those in XR. Fits to the data are shown in Fig. 8 and the results are summarized in Table 1 . The three-layer (air, PS film, and substrate) model with roughness works fine
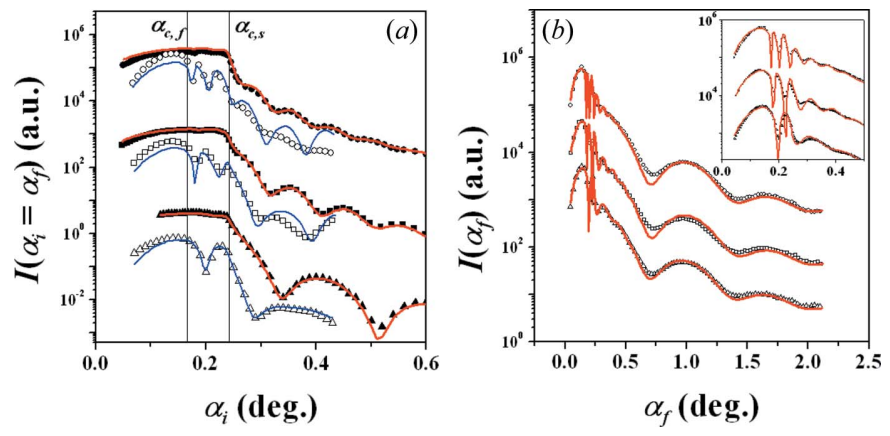

Figure 8

(a) The measured (symbols) data of SM-GISAXS (open) and XR (closed) and their fits for samples I, II and III, from bottom to top. (b) Fits to the data corresponding to the vertical cuts measured at $\alpha_{\mathrm{i}}=0.14^{\circ}$ for the samples. The Kiessig fringes are magnified in the inset. for both XR and GISAXS in this work, optimized with a nonlinear least-squares algorithm.

As seen from Table 1, the differences between the particle positions determined by SM-GISAXS, $d_{\mathrm{GISAXS}}$, and XR, $d_{\mathrm{XR}}$, for the three samples are about $78 \AA$, which is the same as the radius of TMV $\left(R_{\mathrm{TMV}}\right)$. This difference supports our argument on the origin of the Kiessig fringes discussed in $\$ 4.3$ and suggests that the distance $\left(z_{\mathrm{p}}\right)$ between the center of TMV to the surface of PS is about $R_{\mathrm{TMV}}$. Furthermore, it is clear that the low concentration of TMVs makes them invisible to XR. Both $d_{\text {GISAXs }}$ and $d_{\mathrm{XR}}$ can be calculated in a model-independent manner from the Kiessig fringes using the Bragg equation: $d=2 \pi / \Delta q_{z}$, where $\Delta q_{z}$ is the period of the fringes. When the refraction is taken into account, it provides more accurate results. The equation is applicable to both SM-GISAXS and GISAXS, shown in Figs. $8(a)$ and $8(b)$, respectively. For GISAXS, $\left(z_{\mathrm{p}}+t_{\mathrm{f}}\right)=2 \pi / \Delta q_{z} \simeq \lambda /\left(2 \Delta \tilde{\alpha}_{\mathrm{f}}\right)$, where $\tilde{\alpha}_{\mathrm{f}}=$ $\left(\alpha_{\mathrm{f}}^{2}-\alpha_{c}^{2}+2 i \beta\right)^{1 / 2}$ and $\beta$ is the imaginary component of the refractive index, typically of the order of $10^{-9}$ for organic films. For SM-GISAXS, $\left(z_{\mathrm{p}}+t_{\mathrm{f}}\right)=\lambda /\left(2 \Delta \tilde{\alpha}_{\mathrm{i}}\right)$, where $\tilde{\alpha}_{\mathrm{i}}=$ $\left(\alpha_{\mathrm{i}}^{2}-\alpha_{c}^{2}+2 i \beta\right)^{1 / 2}$.

During model fitting of data in Figs. 8(a) and 8(b), we found that SM-GISAXS is sensitive to both film thickness $\left(t_{\mathrm{f}}\right)$ and $z_{\mathrm{p}}$ independently, while the fringes in GISAXS depend on their sum. However, the best fitted film thickness ( $\left.t_{\mathrm{f}, \mathrm{GISAXS}}\right)$ from SM-GISAXS is quite different from that of XR $\left(d_{\mathrm{XR}}\right)$. When we assumed $t_{\mathrm{f}, \mathrm{GISAXS}}=d_{\mathrm{XR}}$ for fitting SM-GISAXS, the positions of the minima in the Kiessig fringes were not correctly reproduced. Although this suggests that the DWBA may not be perfect for SM-GISAXS, it should be kept in mind that GISAXS only detects the area where the TMV exists, whilst $\mathrm{XR}$ probes the entire projected area of the X-ray beam. Interestingly, results shown in Table 1 show the increase in $z_{\mathrm{p}}$ and $\sigma_{\mathrm{p}}$ as a function of the roughness of the film $\left(\sigma_{\mathrm{f}}\right)$, suggesting that TMVs might be sitting on the tips of the rough polymer surface rather than filling the valleys of the roughness completely. The large $\sigma_{\mathrm{p}}$ in Table 1 is presumably due to the overlap of TMVs on each other on the substrate (see the AFM image in Fig. 1).

\subsection{Particle location and GISAXS}

As discussed in $\$ \$ 4.3$ and 4.5 , the Kiessig fringes in GISAXS originate from the distance $t_{\mathrm{f}}+z_{\mathrm{p}}$. However, this is not always true and can either be from $t_{\mathrm{f}}+z_{\mathrm{p}}$ or $t_{\mathrm{f}}$ depending on the distribution of $z_{\mathrm{p}}$. The Kiessig fringes from the distance $t_{\mathrm{f}}+z_{\mathrm{p}}$ disappear as the distribution of $z_{\mathrm{p}}$ becomes broader since the phase terms $\exp \left( \pm i k_{\mathrm{i}} z_{\mathrm{p}}\right)$ and $\exp \left( \pm i k_{\mathrm{f}} z_{\mathrm{p}}\right)$ in the wavefields $\psi_{\mathrm{i}}$ and $\psi_{\mathrm{f}}$ are averaged out. As a result, the amplitudes $T_{\mathrm{i}}, T_{\mathrm{f}}, R_{\mathrm{i}}$ and $R_{\mathrm{f}}$ of the wavefields will determine the period of the Kiessig fringes, where the amplitudes are a function of $t_{\mathrm{f}}$ and do not contain any information on $z_{\mathrm{p}}$. In other words, when the particles are randomly distributed in a film, the phases of a form factor, or $D$ terms in equation (3), are averaged out. Finally, the phase of $T_{\mathrm{f}}$ in equation (3) remains and produces the Kiessig fringes. 
Then, how can we distinguish whether the distribution of $z_{\mathrm{p}}$ is broad and whether the Kiessig fringes are due to the distance $t_{\mathrm{f}}+z_{\mathrm{p}}$ or $t_{\mathrm{f}}$ ? XR measurement in addition to GISAXS is probably the best way to resolve this. However, the characteristic difference in the shapes of the Kiessig fringes of GISAXS from the two different origins also readily enables an estimation of the distribution of $z_{\mathrm{p}}$. To gain some insight on this, we simulated GISAXS at $\alpha_{\mathrm{i}}=0.155^{\circ}$ for various $z_{\mathrm{p}}$; corresponding IAR-GISAXS images are shown in Figs. 9 and 10 , respectively. Simulations were performed for four $z_{\mathrm{p}}$ values: $80 \AA$ as a representative of particles on a film (Fig. 10a), $-80 \AA$ (Fig. 10b) and $-160 \AA$ (Fig. 10c) representing those embedded in a film, and random in a film (Fig. 10d). In Fig. 9, the Kiessig fringes in the top three curves for particles at defined positions appear larger than the WGR, whereas that in the bottom curve shows up only in the WGR. There are some discrepancies in the shape of the fringes as well. Since the Kiessig fringes originate from the intensity of the wave-

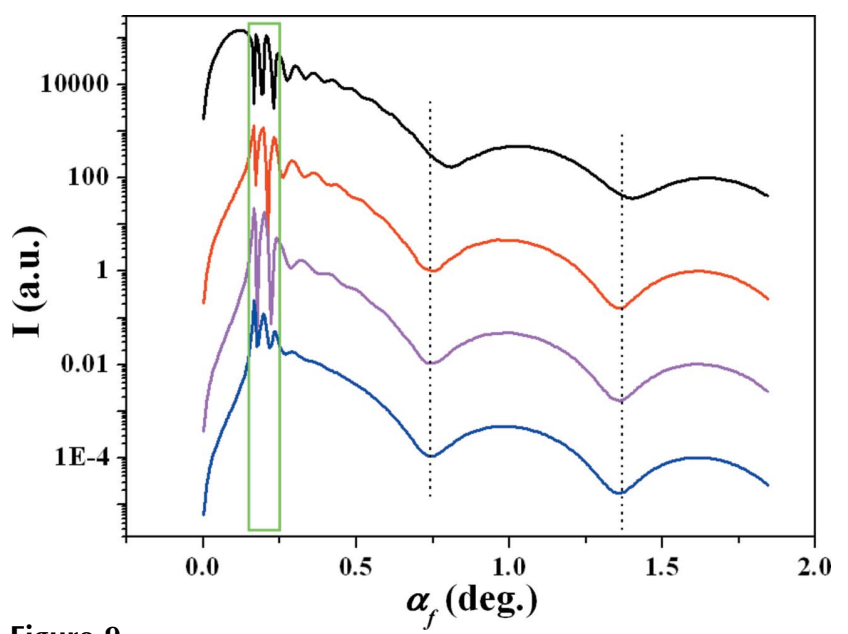

Figure 9

GISAXS simulation for spherical particles of radius $R=80 \AA$ located at $z_{\mathrm{p}}=80 \AA$ (black), $-80 \AA$ (red), $-160 \AA$ (purple), and randomly (blue) embedded in a film on a Si substrate. The wave-guiding region (WGR) is shaded. The form-factor minima, M1 and M2, are denoted with broken lines. The representation is the same as in Figs. 5 and 7.
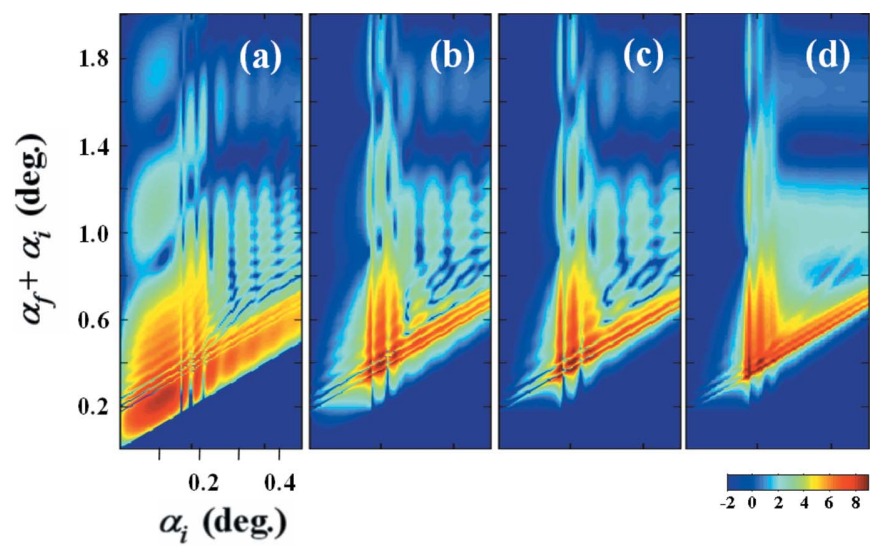

Figure 10

Calculated IAR-GISAXS images at $0.01<\alpha_{\mathrm{i}}<0.45^{\circ}$ for the particles described in Fig. 9; $z_{\mathrm{p}}=80 \AA(a),-80 \AA(b),-160 \AA(c)$, and randomly embedded in a film on a Si substrate $(d)$. field $\left(\psi_{\mathrm{f}}\right)$, for the defined position of the sample, they likely have a sinusoidal shape. While that for the randomly distributed one is from $T_{\mathrm{f}}$ or $R_{\mathrm{f}}$, it seems that the peak minima cannot reach 0 . Note that intensities at the minima of the fringes for the latter are higher than the form-factor curve in Fig. 9. The simulation of IAR-GISAXS images shown in Fig. 10 nicely demonstrates the effect of the particle position on GISAXS. As the particle position is closer to the surface of the substrate, the number of Kiessig fringes in both sides of $\alpha_{\mathrm{i}}$ and $\alpha_{\mathrm{f}}$ reduces: about $4,3.5$ and 3 fringes are seen along the $\alpha_{\mathrm{i}}$ axis in IAR-GISAXS images for the particles located at $z_{\mathrm{p}}=80$, -80 and $-160 \AA$, respectively. In addition, it can be readily recognized from the IAR-GISAXS image whether the particles sit on a film (Fig. 11a) or are embedded in the film. When the particle is completely embedded in the PS film, scattering almost disappears in the areas of $\alpha_{\mathrm{i}}<\alpha_{\mathrm{c}, \mathrm{f}}$ and $\alpha_{\mathrm{f}}<\alpha_{\mathrm{c}, \mathrm{f}}$ (see Figs. 10b-10d compared with Fig. 11a) (Lee, Park et al., 2005; Babonneau et al., 1999).

Based on the parameters extracted from the fits to the Kiessig fringes shown in Fig. 8 and the form factor (Lee, Lo et al., 2007), we calculated the IAR-GISAXS images shown in Figs. $11(b)$ and $11(d)$ to compare with the measured data in Figs. 11(a) and 11(c). As demonstrated in the previous simulations, the Kiessig fringes exist along both sides of the $\alpha_{\mathrm{f}}+\alpha_{\mathrm{i}}$ and $\alpha_{\mathrm{i}}$ axes. Since their periods are a function of the distance $t_{\mathrm{f}}$ $+z_{\mathrm{p}}$, the number of vertical Kiessig fringes in Fig. 11(a) for the bio-particles on a thinner PS film is less than that in Fig. 11(c). These fringes are clearly visible, even at $\alpha_{\mathrm{i}}>\alpha_{\mathrm{c}, \mathrm{s}}$, as shown with short arrows, indicating that the TMVs sit at well defined positions along $z$, and are not distributed randomly. Interestingly, the minima in the vertical Kiessig fringes (see the dotted line arrows in Figs. $11 a$ and 11c) are curved, which is due to the interference between the form factor and the Kiessig fringes. These curved Kiessig fringes again demonstrate that the standing-wave concept applied in equations (4)-(6) is inappropriate for our work dealing with particles with a radius of about $80 \AA$. The interference between the form factor and

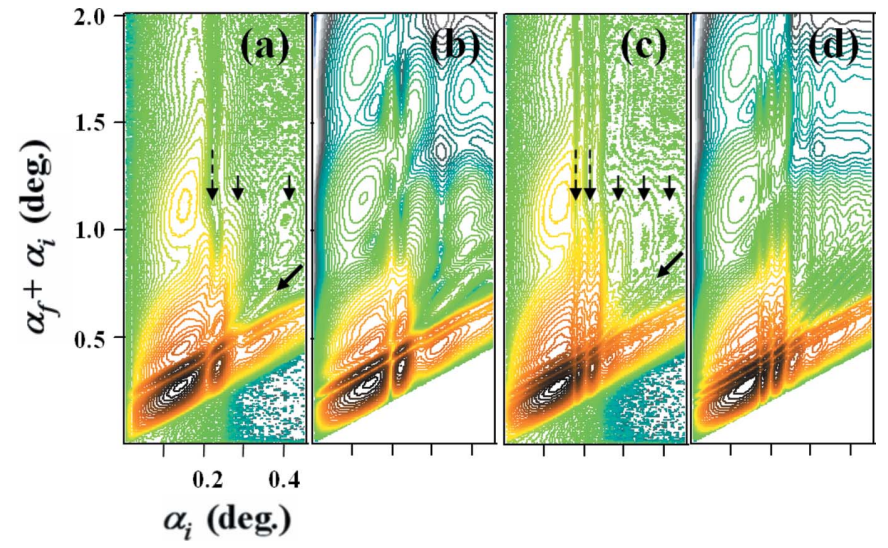

Figure 11

The measured, $(a)$ and $(c)$, and the calculated, $(b)$ and $(d)$, IAR-GISAXS at $2 \theta_{\mathrm{f}}=0.15^{\circ}$ for sample I, $(a)$ and $(b)$, and III, $(c)$ and $(d)$. Short and long solid arrows designate the Kiessig fringes and SM line at $\alpha_{\mathrm{i}}>\alpha_{\mathrm{cs}, \mathrm{s}}$ and $\alpha_{\mathrm{f}}>\alpha_{\mathrm{c}, \mathrm{s}}$. Broken arrows show the location of the minima in the Kiessig fringes, which are bent by the interference with the form-factor scattering of the TMV. The color scale is identical to that of Fig. 2. 
the Kiessig fringes produces new fringes parallel to the $\alpha_{\mathrm{i}}=\alpha_{\mathrm{f}}$ line (or SM line) as shown with thick short arrows in Figs. 11(a) and 11(c), which also would not be expected for very small particles. As shown in $\S 4.5$, the Kiessig fringes on the SM line and the complicated interference pattern around it are sensitive to both $z_{\mathrm{p}}$ and $t_{\mathrm{f}}$ independently, whereas the Kiessig fringes along either the $\alpha_{\mathrm{i}}$ or the $\alpha_{\mathrm{f}}+\alpha_{\mathrm{i}}$ axis are sensitive to their sum. Hence, they could be useful as a fingerprint to determine $z_{\mathrm{p}}$ accurately from the sum determined from the Kiessig fringes.

\section{Conclusion}

In summary, we have resolved the origin of various interferences in GISAXS due to the multiple scattering generated by two direct beams: incident and reflected. The multiple scattering effects distort the form-factor scattering and produce the Kiessig fringes. We have shown how these effects are useful to determine particle positions. In addition, we provide a novel concept that enables the use of kinematic theory for the analysis of GISAXS data containing multiple scattering, called the single-beam approximation. The imaginary single beam is assumed to propagate parallel to the substrate surface. The distance between the center of a particle and the surface of a substrate can be calculated from the Kiessig fringes using the Bragg equation.

As a demonstration, we studied tobacco mosaic virus (TMV) adhered on a spin-coated PS film on a Si wafer using both XR and GISAXS. GISAXS measured as a function of the incident angle, termed incident-angle-resolved GISAXS, reveals the interference between the form factor of a particle and the standing waves. Simulation demonstrates that DWBA theory could successfully reproduce the phenomenon. It turns out that the particles are invisible to XR due to their low coverage on a surface; however, their positions and positional distribution are well resolved by the Kiessig fringes in GISAXS and IAR-GISAXS, indicating that GISAXS/IARGISAXS is sensitive to both the position and orientational distribution of the particles, while XR is sensitive only to the interfaces. For both techniques, the modified Bragg equation can be used to extract the distance between the center of a particle and the surface of a substrate.

To conclude, GISAXS measurement at various incident angles is useful to reduce the smearing effects caused by multiple scattering (see \$4.2) and to readily extract positional information for nanoparticles (see §4.6). Such a measurement can also help to determine the sign of the scattering envelope, as discussed in $\S 4.2$.

This work benefited by the use of the APS and other facilities at the Argonne National Laboratory supported by the US Department of Energy, Office of Science, Office of
Basic Energy Sciences, under Contract No. DE-AC0206CH11357. QW would like to acknowledge the financial support from the ARO-MURI program and the W. M. Keck Foundation.

\section{References}

Babonneau, D., Naudon, A., Thiaudière, D. \& Lequien, S. (1999). J. Appl. Cryst. 32, 226-233.

Bedzyk, M. J., Bommarito, G. M. \& Schildkraut, J. S. (1989). Phys. Rev. Lett. 62, 1376-1379.

Busch, P., Rauscher, M., Smilgies, D.-M., Posselt, D. \& Papadakis, C. M. (2006). J. Appl. Cryst. 39, 433-442.

Chang, S.-L. (2004). X-ray Multiple-Wave Diffraction. Berlin: Springer.

Guinier, A. \& Fournet, G. (1955). Small-Angle Scattering of X-rays. New York: John Wiley.

Holy, V. \& Baumbach, T. (1994). Phys. Rev. B, 49, 10668-10676.

Kapp, D. S. \& Wainfan, N. (1965). Phys. Rev. 138, A1490-A1495.

Kiessig, H. (1931). Ann. Physik, 10, 769-788.

Lazzari, R. (2002). J. Appl. Cryst. 35, 406-421.

Lee, B., Lo, C.-T., Winans, R. E., Thiyagarajan, P., Niu, Z. \& Wang, Q. (2007). Langmuir, 23, 11157-11163.

Lee, B., Park, I., Park, H., Lo, C.-T., Chang, T. \& Winans, R. E. (2007). J. Appl. Cryst. 40, 496-504.

Lee, B., Park, I., Yoon, J., Park, S., Kim, J., Kim, K.-W., Chang, T. \& Ree, M. (2005). Macromolecules, 38, 4311-4323.

Lee, B., Yoon, J., Oh, W., Hwang, Y., Heo, K., Jin, K. S., Kim, J., Kim, K.-W. \& Ree, M. (2005). Macromolecules, 38, 3395-3405.

Lee, D. R., Hagman, A., Li, X., Narayanan, S., Wang, J. \& Shull, K. R. (2006). Appl. Phys. Lett. 88, 153101.

Levine, J. R., Cohen, J. B., Chung, Y. W. \& Georgopoulos, P. (1989). J. Appl. Cryst. 22, 528-532.

Narayanan, S., Lee, D. R., Guico, R. S., Sinha, S. K. \& Wang, J. (2005). Phys. Rev. Lett. 94, 145504.

Parratt, L. G. (1954). Phys. Rev. 95, 359-369.

Rauscher, M., Salditt, T. \& Spohn, H. (1995). Phys. Rev. B, 52, 1685516863.

Renaud, G., Lazzari, R., Revenant, C., Barbier, A., Noblet, M., Ulrich, O., Leroy, F., Jupille, J., Borensztein, Y., Henry, C. R., Deville, J.-P., Scheurer, F., Mane-Mane, J. \& Fruchart, O. (2003). Science, 300, 1416-1419.

Roe, R.-J. (2000). Methods of X-ray and Neutron Scattering in Polymer Science. New York: Oxford University Press.

Roth, S. V., Burghammer, M., Riekel, C., Müller-Buschbaum, P., Diethert, A. \& Panagiotou, P. (2003). Appl. Phys. Lett. 82, 19351937.

Sanyal, M. K., Sinha, S. K., Gibaud, A., Huang, K. G., Carvalho, B. L., Rafailovish, M., Sokolov, J., Zhao, X. \& Zhao, W. (1993). Europhys. Lett. 21, 691-696.

Sinha, S. K., Sirota, E. B., Garoff, S. \& Stanley, H. B. (1988). Phys. Rev. B, 38, 2297-2311.

Stein, G. E., Kramer, E. J., Li, X. F. \& Wang, J. (2007). Macromolecules, 40, 2453-2460.

Vartanyants, I. A., Grigoriev, D. \& Zozulya, A. V. (2007). Thin Solid Films, 515, 5546-5552.

Vineyard, G. H. (1982). Phys. Rev. B, 26, 4146-4159.

Wang, J., Bedzyk, M. J., Penner, T. L. \& Caffrey, M. (1991). Nature (London), 354, 377-380.

Wang, Y., Watkins, E., Ilavsky, J., Metroke, T. L., Wang, P., Lee, B. \& Schaefer, D. W. (2007). J. Phys. Chem. B, 111, 7041-7051. 